\section{Market for superconductors is expected to soar by 2020}

Tokyo. Industrialists in Japan, Europe and the United States involved in superconductor research believe that, given sufficient government support for research and development, the market for superconductors could rise from its present level of US $\$ 1.5$ billion to $\$ 150-\$ 200$ billion by 2020 . The projections, made at the second International Superconductivity Industry Summit (ISIS) held in Hakone by Mount Fuji in

\section{The promise of superconductors...}

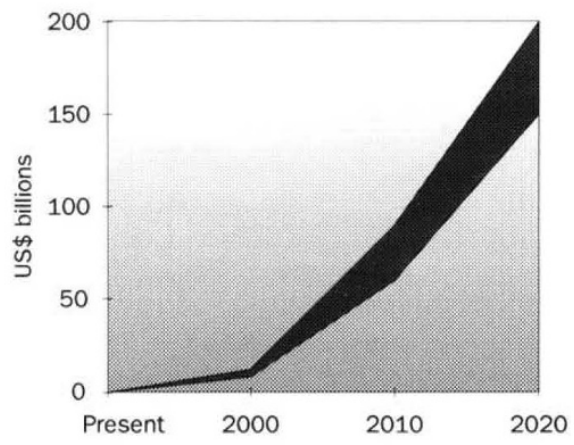

$\ldots$ and their uses

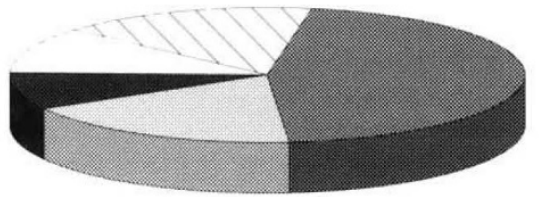

Electronics $\square$ Energy $\square$ Transport
$\square$ Medical $\square$ Others

Japan, are intended to persuade national governments to continue their support for superconductor research beyond the several hundred million dollars a year being spent in the three regions.

ISIS, made up of industrialists and researchers from Japan's International Superconductivity Technology Center (ISTEC), the US Council on Superconductivity for American Competitiveness (CSAC) and the recently formed Consortium of European Companies determined to use Superconductivity (CONECTUS), made the projections on the basis of information from companies in each region. Projections were converted to a worldwide figure on the basis of gross national product. Helmut Piel of the Bergische Universität Gesamthochschule Wuppertal in Germany, who with Sir Martin Wood of Britain's Oxford Instruments helped to create CONECTUS in March, says that he was pleasantly surprised at the consensus on both the projected size of the total market and the relative share of individual applications.

The present market of $\$ 1.5$ billion consists entirely of products made from conventional low-temperature superconductors, in particular magnetic resonance imaging devices for medical use. But by 2020 , products made with high-temperature superconductors will comprise the dominant share, predicts CSAC representative Alan Lauder of DuPont.

ISIS members admit that there are uncertainties in their estimates. "Superconductivity may well develop a major business through an unexpected technological breakthrough in an unrelated field", according to ISIS, in the same way as the market for semiconductor lasers grew rapidly because of compact-disc players. And the discovery of superconductors with even higher critical temperatures (Tc) could result in even larger markets, says Lauder.

But much of this growth will depend on government policies. Achieving the projected market size will require an investment of "hundreds of millions of dollars over the next 30 years", says Lauder, some of which must come from governments.

In the United States, that investment has grown from $\$ 40$ million in 1986 to $\$ 250$ million in 1992, much of it for lowtemperature superconducting magnets for the Superconducting Super Collider (SSC). Similarly, Japan increased its spending dramatically after the discovery of hightemperature superconductors in 1986 to the present level of about $¥ 18$ billion (US\$162 million). Investment in Europe is on the same order of magnitude as in Japan, says Gordon Donaldson of the University of Strathclyde in Scotland.

But the future does not look so bright. Britain's Department of Trade and Industry has just terminated a programme that provided about $£ 8$ million (US\$12 million) a year, and Japan's Science and Technology Agency (STA) will reduce its support for an important project in the field from $¥ 2.1$ billion this year to no more than $¥ 1.3$ billion next year, although STA officials hope to start a second phase in 1995. ISIS members in the United States admit that one of the purposes of the market projection is to persuade the US government to continue its investment in superconductor research regardless of the fate of the SSC.

Next year, at a meeting in Europe, ISIS plans to arrange practical demonstrations of superconductor devices. The first projects are likely to be national ones, says Piel of Germany, followed by international collaboration.

\section{China struggles with home-grown 'Gordon conference'}

Beijing. Five years ago, the Chinese State Science and Technology Commission started an annual seminar modelled on the Gordon Research Conferences held every summer in New Hampshire in the United States. The seminars, called the Xiangshan ('Fragrant hills') Science Conference after a picturesque site in the foothills west of Beijing, were intended to foster the free exchange of ideas among different disciplines.

Last month, as prominent scientists such as Zhou Guang-Zhao, president of the Chinese Academy of Sciences, gathered at Xiangshan, it was clear that the concept has failed to take root. The traditions of science in China militate against such a free-flowing debate among those in different fields and of different rank, and the result, according to participants, has been discussions that can charitably be described as dull and nonsubstantive.

The rules are not to blame. Participants are told to speak their minds and government officials, in a remarkable departure from common practice, take part only as individual scientists. Those who have attended say that the procedures are quite helpful.

But Chinese scientists are not accustomed to open debate. Public disagreements are regarded as rude in a culture that reveres seniority. In addition, without an effective system to protect intellectual property, Chinese scientists are reluctant to talk about new findings for fear that others will steal their ideas. Cases of alleged infringement fill the courts, and even groups working on similar topics within the same institution refuse to share data. The Chinese refer to such communications problems as "internal friction", but efforts by government officials to bring about change have had little effect.

Part of the problem rests with training. Educated in a university system drawn from the rigid Soviet model, most researchers have a very narrow view of science and a limited knowledge of fields outside their speciality. As a result, they can contribute little to a discussion that ranges across several disciplines. Instead, participants choose topics that do not require specialized knowledge, for example 'science in the next century', and in which they are unlikely to be proved wrong.

Despite questions about the value of the conferences, the state commission seems determined to support them. Zhou GuangZhao would like to see them continue, and the commission has already allocated money for a sixth meeting next year. Given the dissatisfaction among scientists, however, organizers may have to face the fact that good intentions alone are not enough to sustain this annual gathering. You Qin LI 